\title{
Effects of Topical Brinzolamide on Infantile Nystagmus Syndrome Waveforms: Eyedrops for Nystagmus
}

\author{
Louis F. Dell'Osso, PhD, Richard W. Hertle, MD, R. John Leigh, MD, \\ Jonathan B. Jacobs, PhD, Susan King, BS, Stacia Yaniglos, OD
}

\begin{abstract}
Background: Recent advances in infantile nystagmus syndrome (INS) surgery have uncovered the therapeutic importance of proprioception. In this report, we test the hypothesis that the topical carbonic anhydrase inhibitor (CAI) brinzolamide (Azopt) has beneficial effects on measures of nystagmus foveation quality in a subject with INS. Methods: Eye movement data were taken, using a highspeed digital video recording system, before and after 3 days of the application of topical brinzolamide 3 times daily in each eye. Nystagmus waveforms were analyzed by applying the eXpanded Nystagmus Acuity Function (NAFX) at different gaze angles and determining the longest foveation domain (LFD) and compared to previously published data from the same subject after the use of a systemic CAI, contact lenses, and convergence and to other subjects before and after eye muscle surgery for INS.

Results: Topical brinzolamide improved foveation by both a $51.9 \%$ increase in the peak value of the NAFX function (from 0.395 to 0.600 ) and a $50 \%$ broadening of the NAFX vs Gaze Angle curve (the LFD increased from $20^{\circ}$ to $30^{\circ}$ ). The improvements in NAFX after topical brinzolamide were equivalent to systemic acetazolamide or eye muscle surgery and were intermediate between those of soft contact lenses or convergence. Topical brinzolamide and contact lenses had equivalent LFD improvements and were less effective than convergence.
\end{abstract}

Daroff-Dell'Osso Ocular Motility Laboratory (LFD, RJL, JBJ, SK, SY), Louis Stokes Cleveland Department of Veterans Affairs Medical Center and CASE Medical School, Cleveland, Ohio; Departments of Neurology (LFD, RJL, JBJ) and Biomedical Engineering (LFD, RJL, JBJ), Case Western Reserve University and University Hospitals Case Medical Center, Cleveland, Ohio; Department of Ophthalmology (RWH), Children's Hospital Medical Center of Akron, Akron, Ohio; and Department of Ophthalmology (RWH), Northeastern Ohio Universities College of Medicine, Rootstown, Ohio. Dr L. F. Dell'Osso is currently professor emeritus of neurology.

Supported in part by the Office of Research and Development, Medical Research Service, Department of Veterans Affairs.

The authors report no conflicts of interest.

Address correspondence to Louis F. Dell'Osso, PhD, Daroff-Dell'Osso Ocular Motility Laboratory, Louis Stokes Cleveland Department of Veterans Affairs Medical Center, 10701 East Boulevard, Cleveland, OH 44106; E-mail: lfd@case.edu
Conclusions: In this subject with INS, topical brinzolamide resulted in improved-foveation INS waveforms over a broadened range of gaze angles. Its therapeutic effects were equivalent to systemic CAl. Although a prospective clinical trial is needed to prove efficacy or effectiveness in other subjects, an eyedrops-based therapy for INS may emerge as a viable addition to optical, surgical, behavioral, and systemic drug therapies.

Journal of Neuro-Ophthalmology 2011;31:228-233

doi: 10.1097/WNO.0b013e3182236427

(c) 2011 by North American Neuro-Ophthalmology Society

I nfantile nystagmus syndrome (INS) is an ocular motor oscillation with well-defined clinical characteristics and eye movement waveforms $(1,2)$. In some individuals, it is inherited (2), but its pathogenesis remains unknown. Current therapy may damp INS oscillations and result in improved foveation, but the nystagmus persists despite the ocular motor system's best efforts to improve foveation periods (3). Surgical (4-6), optical (7), and pharmaceutical (8-10) therapeutic approaches have been successfully employed to improve INS waveforms and expand the range of gaze angles with higher visual acuity $(11,12)$. We have recently studied the beneficial effects of systemic acetazolamide on INS simulated in a behavioral ocular motor system model, which suggested that the drug may have also had peripheral effects (13). The present report documents the results of applying topical brinzolamide eyedrops to the same INS subject that showed improvements with systemic acetazolamide and provides evidence for the hypothesis that an eyedrops-based therapy for INS is possible.

\section{METHODS}

\section{Recording}

We used a digital video system (EyeLink II; SR Research, Mississauga, Ontario, Canada) for the eye movement 
recordings. The system had a linear range of $\pm 30^{\circ}$ horizontally and $\pm 20^{\circ}$ vertically. System sampling frequency was $500 \mathrm{~Hz}$, and gaze position accuracy error was $0.5^{\circ}-1^{\circ}$ on average. The data from this system were digitized at 500 $\mathrm{Hz}$ with 16-bit resolution. The EyeLink signal from each eye was calibrated with the other eye behind cover to obtain accurate position information; the foveation periods were used for calibration.

\section{Subject and Protocol}

This study was approved by our institutional review board, and written consent was obtained from the subject. The patient was a 68-year-old man with INS. His nystagmus waveforms have been well documented over a period of approximately 50 years, and their characteristics have remained constant and proved to be representative of others with INS. Because subjects have no voluntary control of their INS waveform characteristics (i.e., they cannot change their INS waveforms or improve their foveation qualities), subsequent testing or knowledge of the experiment does not affect the data. Stress can only worsen INS, and our stressfree testing environment (requiring merely looking at lightemitting diodes (LEDs) or laser spots) allows for accurate intra- and intersubject comparisons. Our subject had a convergence "null" and normally wore either 7 prism diopters (PD) base-out prisms added to his spectacles (right eye: $+3.00-2.50 \times 150^{\circ}$ and left eye: $+3.50-2.75 \times 20^{\circ}$ ) or contact lenses with no added prism. Best-corrected distance visual acuities were 20/25 with prisms and 20/40 with contact lenses. For fixation targets, we used small LEDs and reflected laser spots that yield the same ocular motor data with or without refraction. The subject was seated in a chair with a headrest and a chin stabilizer, far enough ( $>5$ feet) from the stimulus screen to prevent convergence effects. At this distance, the target subtended less than $0.1^{\circ}$ of visual angle. The room light was turned off during the recording. Each trial tested fixation of horizontal targets from $30^{\circ}$ left gaze to $30^{\circ}$ right gaze, in $5^{\circ}$ steps. Our standard testing paradigm consisted of 2 trials, one in each horizontal direction, with the target remaining at each gaze angle for 5 seconds during both stepping out laterally and returning to primary position. Subtracting the required target acquisition times allowed approximately 2-3 seconds of steady fixation during each interval. eXpanded Nystagmus Acuity Function (NAFX) values (see below) were calculated during steady fixations and averaged. This resulted in a small number of NAFX values at each gaze angle that we have found to be historically close to each other in value. Gathering and analyzing such data are based on reallife acquisition and identification of new targets in the visual field.

The subject was given 1 drop of topical brinzolamide (Azopt) 3 times daily in each eye on days 1-3, after which the eyedrops were discontinued. The subject had eye movement recordings twice on day 1 ( 1 hour and 5 hours after the first administration of brinzolamide) and once on days 2-5 and 12. Ocular and systemic evaluations were performed during the 17-day study period [intraocular pressure (IOP) was first recorded 5 days prior to administration of brinzolamide] to check for adverse events. For comparisons of therapeutic effectiveness, we retrieved data from a previous study of the effects of systemic acetazolamide on the same subject (14).

\section{Analysis}

All the analyses were performed in the MATLAB environment (The MathWorks, Inc, Natick, MA) using OMLAB software (OMtools, downloadable from http://www.omlab.org). Eye position was sampled directly; it was prefiltered using a lowpass filter with a cutoff frequency of $20 \mathrm{~Hz}$ to reduce the noise while minimally affecting the foveation periods. The $20-\mathrm{Hz}$ value is 5 times the maximum frequency present in foveation periods $(0-2 \mathrm{~Hz})$ and still allows separation of foveating and braking saccades from those periods. Analysis was always done on the fixating eye. Segments with inattention or blinking were discarded.

We analyzed the data using the NAFX (15). The NAFX is a mathematical function containing the following waveform parameters: duration of foveation period, standard deviations of main foveation periods and velocities, and number of cycles in a fixation interval. In the OMtools software, we use the NAFX's graphical user interface for data selection and analysis (details can be obtained from http://www.omlab.org/OMLAB_page/ Teaching/Using_NAFX.html) (16). The NAFX provides an objective and repeatable measure of nystagmus foveation quality that accurately predicts the best-corrected visual acuity possible for subjects without afferent visual system defects, regardless of the eye movement recording system, the nystagmus type, and waveforms (15). We averaged the NAFX values obtained at each gaze angle.

The longest foveation domain (LFD) $(5,11)$ is the range of gaze angles in which the subject's NAFX stays above $90 \%$ of the NAFX vs Gaze Angle curve's peak value. Thus, the LFD is a measure of the broadness of the NAFX vs Gaze Angle curve, i.e., the INS subject's high-foveation quality field.

\section{RESULTS}

\section{Subject Data}

During fixation of a distant target prior to the administration of topical brinzolamide, the subject's peak NAFX was 0.385 at $2^{\circ}$ left gaze and the breadth of the NAFX vs Gaze Angle curve given by the LFD function was $20^{\circ}$ (Fig. 1). Also shown for comparison are the data for contact lenses and when converged at $60 \mathrm{PD}$. During the 3 days of topical brinzolamide, the NAFX peaks were $0.440,0.553$, 0.540 , and 0.680 , respectively, for days $2,3,4$, and 5 , with LFDs of $46^{\circ}, 35^{\circ}, 30^{\circ}$, and $26^{\circ}$, respectively. All treatments (contact lenses, topical brinzolamide, and convergence) 
NAFX vS GAZE ANGLE

(Contact Lenses, Brinzolamide, Convergence)

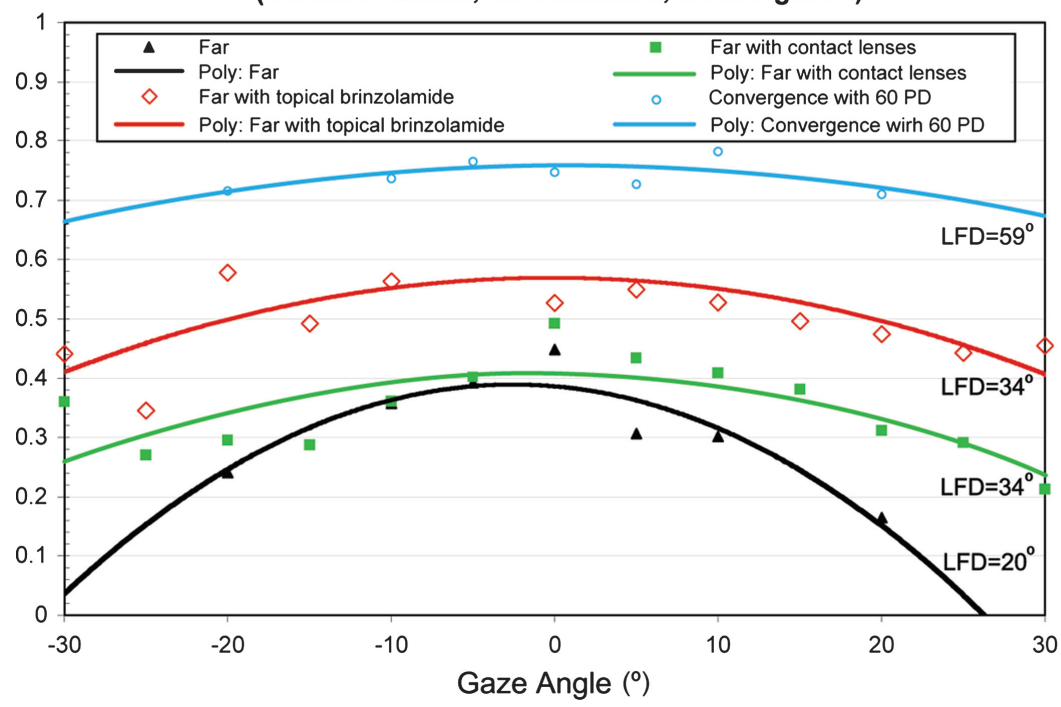

FIG. 1. NAFX vs Gaze Angle plots before administration of topical brinzolamide, with contact lenses, during convergence, and on day 4 with topical brinzolamide. Measured visual acuity values are those determined clinically via a Snellen chart to correlate with NAFX values. Data of convergence and contact lenses are from prior studies ( 7,11$)$ of the same subject. Poly, polynomial fit curve; positive gaze angle, gaze right; negative gaze angle, gaze left.

broadened the pretreatment curve. In addition, topical brinzolamide and convergence also raised its peak value.

Figure 2 shows the damping of the subject's waveforms after topical brinzolamide. It should be noted that although the post-brinzolamide INS waveforms have noticeably lower amplitudes at $0^{\circ}$ and $\pm 15^{\circ}$, they do not at the other gaze angles shown. This finding and the lack of correspondence of INS amplitude to visual acuity reinforce the need for functions like the NAFX and LFD to identify and quantify meaningful therapeutic improvements. For example, the percentage increases in the NAFX at $0^{\circ}$ (where there is an

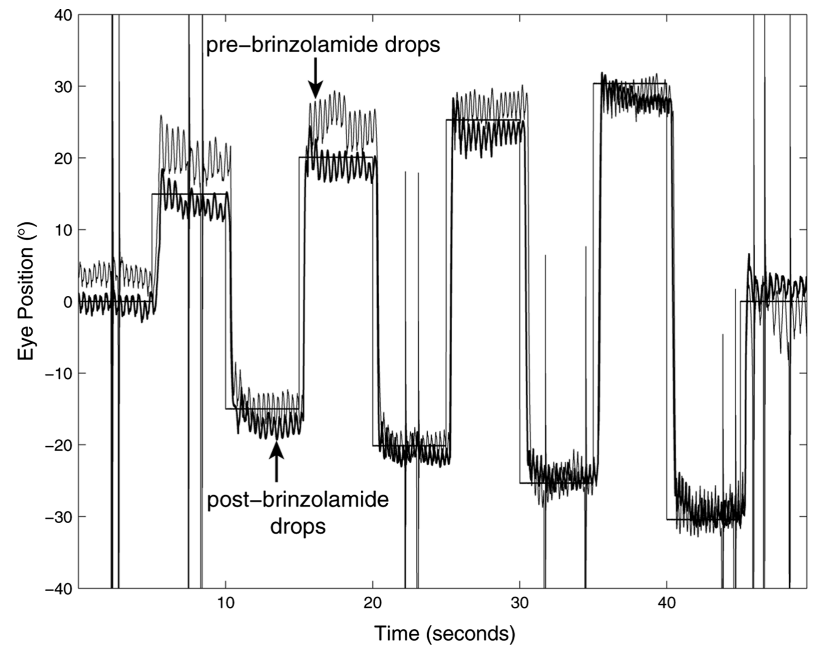

FIG. 2. Example of INS waveforms prior to (gray line) and following (black line) the use of brinzolamide eyedrops. amplitude difference) and $20^{\circ}$ (where there is a minimal amplitude difference) are $36.7 \%$ and $184 \%$, respectively.

The percent improvements in the NAFX peaks were $13 \%, 42 \%, 38 \%$, and $74 \%$ for the effects of 4 days of topical brinzolamide. The corresponding percent improvements in the LFD were $130 \%, 75 \%, 50 \%$, and $30 \%$. As with all other therapies, topical brinzolamide produced much greater percent NAFX improvements in lateral gaze $\left(550 \%, 713 \%\right.$, and $738 \%$, respectively, at $\left.-30^{\circ}\right)$. The steady-state values of posttherapy peak NAFX and LFD were approximately $0.6^{\circ}$ and $30^{\circ}$, respectively, corresponding to increases in these measures of $51.9 \%$ and $50 \%$.

\section{Comparison to Contact Lenses, Convergence, and Tenotomy and Reattachment}

In addition to comparing the therapeutic effects of topical brinzolamide on peak NAFX and LFD to contact lenses and convergence therapies, we also compared each to the estimated effects of tenotomy and reattachment (T\&R) eye muscle surgery based on the data from 22 previously studied patients (12). Figure 3A demonstrates that brinzolamide raised the peak NAFX by an amount equivalent to that estimated for the T\&R procedure and was superior to contact lenses but not as efficacious as convergence. The $50 \%$ LFD improvement for topical brinzolamide was slightly less than for systemic acetazolamide, contact lenses, and the estimate for surgery; all were less than for convergence (Fig. 3B). The curves in Figure 3 are the estimated percent increases in peak NAFX and LFD for the T\&R procedure based on the results from INS patients who 

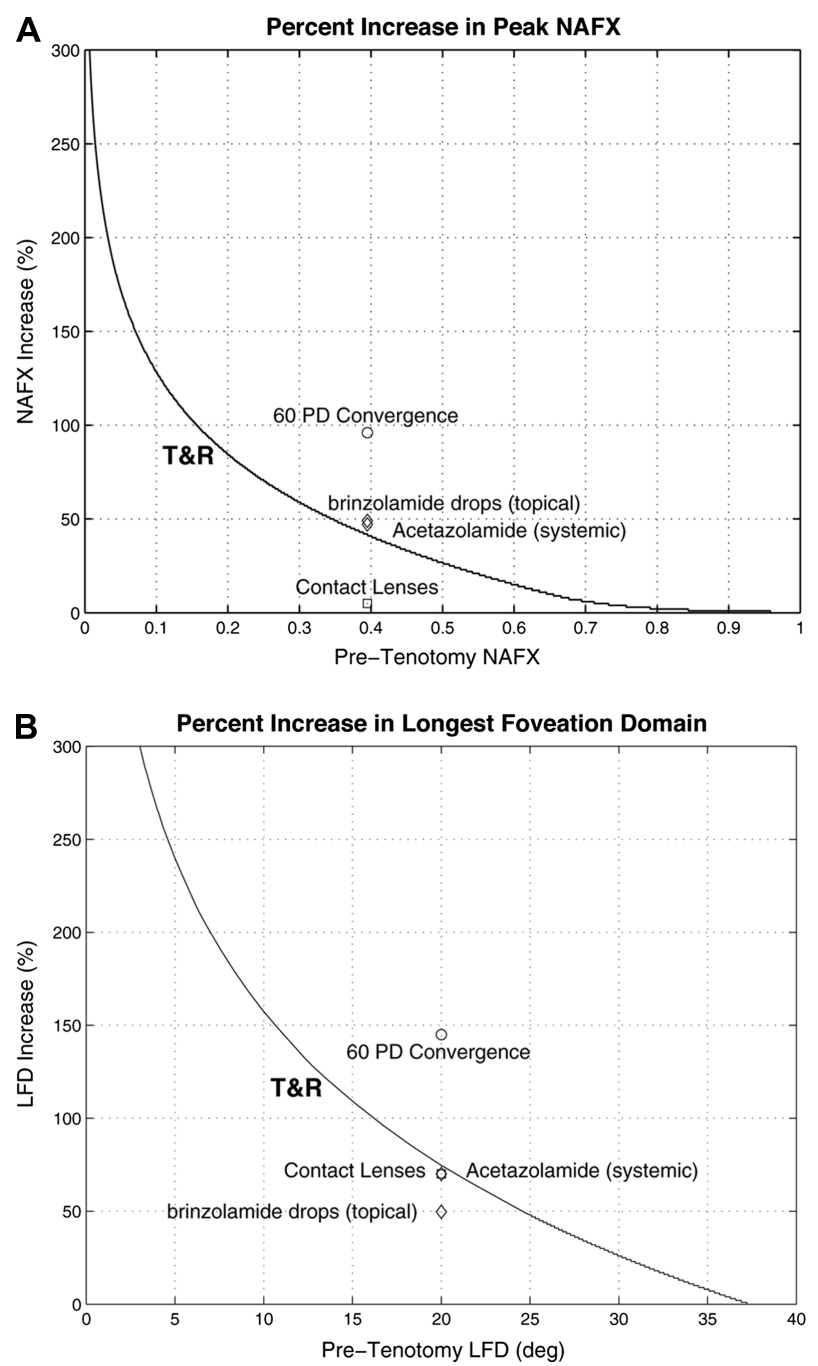

FIG. 3. Comparative plots of percent increases in peak NAFX vs pretherapy NAFX $(\mathbf{A})$ and percent increases in LFD (B) vs pretherapy LFD during fixation of far targets with contact lenses, with topical brinzolamide, and during convergence at 60 PD. The estimated improvement curves were fitted to data from a study on the effects of T\&R surgery ( 7 ), and data points from prior publications are taken from studies of convergence (11), contact lenses (7), and systemic acetazolamide (13).

underwent that procedure. Once the pre-T\&R NAFX and LFD values are calculated, the curves allow estimation of the post-T\&R improvements independent of the existence of any afferent sensory deficits or of the age of the patient. Given the pre-T\&R measured visual acuity, we can estimate the post-T\&R measured acuity. This cannot be done from clinical observations or tests.

\section{Side Effects of Topical Carbonic Anhydrase Inhibitor}

We monitored the effects of the eyedrops on IOP of our patient. The pretherapy IOP was $16 \mathrm{~mm} \mathrm{Hg}$, which was reduced to $12 \mathrm{~mm} \mathrm{Hg}$ during the trial and had returned to $14.5 \mathrm{~mm} \mathrm{Hg}$ by day 5 (2 days after the last eyedrops). There were no adverse systemic events as a result of the 3-day course of topical brinzolamide.

\section{DISCUSSION}

\section{Rationale for the Use of Topical Carbonic Anhydrase Inhibitor in the Treatment of Infantile Nystagmus Syndrome}

Dell'Osso et al $(17)$ and Hertle et al $(4,18)$ have demonstrated that eye muscle T\&R alone had salutary effects on nystagmus amplitude and velocity in dogs with nystagmus and in 2 human trials in patients with INS. A hypothesis evolved that $T \& R$ altered proprioceptive structures in the eye muscle tendon at its insertion on the globe (enthesis) that favorably affected the nystagmus oscillation. Hertle et al $(19)$ and Büttner-Ennever et al $(20,21)$ have studied the anatomical and physiological properties of enthesial neurons. These neurons probably provide feedback that assists with ocular alignment and stabilization. Surgical disruption of enthesis in patients with INS results in long-standing beneficial effects on nystagmus and visual function $(4,5,18,22)$. This may be due to a reduction of small signal gain of the ocular motor plant by interfering with enthesial proprioceptive tension control. Enthesial nerves are probably palisade-type non-twitch motoneurons and are likely involved in modulating the gain of sensory feedback from the eye muscles analogous to the gamma motoneurons, which control the gain of proprioceptive feedback in skeletal muscles.

Carbonic anhydrase (CA) may play an important role in the neurochemical functioning of the membrane potentials of enthesial nerve endings as it does in other sensory systems (23-27). Numerous CA-positive neurons have been found in the trigeminal and geniculate ganglia as well as in the mescencephalic trigeminal nucleus (28-30). There is evidence that CA participates in the response of sensory stretch receptors of the trigeminal nerve and its nerve endings. A functioning CA system may be involved in facilitating enthesial neuronal feedback to central ocular motor areas and continuing to enhance the developmentally disturbed circuit, thereby resulting in potentiating the ocular oscillation of INS. A carbonic anhydrase inhibitor (CAI) may interfere with the sodium-potassium ATPase membranebound system, thus interrupting enthesial neurophysiology (analogous to surgery) and creating a damped circuit, resulting in improvement in the ocular oscillation and enhanced visual function.

\section{Underlying Mechanisms}

Ocular motility studies have shown that INS has characteristic waveforms with typical variations depending on gaze and vergence angles. This is true regardless of associated 
afferent visual abnormalities. INS instability is indistinguishable between the patient populations and has the same direct functional cause of loss of pursuit-system damping (31-33). Although the direct functional cause is the same in individual patients, the precipitating factors and other mechanisms (e.g., effects of vestibulo-ocular imbalance on the position and breadth of the null area, the thresholds at which pendular waveforms convert to jerk) probably differ. Therapies may be directed: 1) afferently to alleviate the visual condition that interfered with damping calibration (34-36), 2) centrally at the cells responsible for damping miscalibration (10-12), or 3) peripherally to reduce the effects of the underlying instability (e.g., vergence prisms, contact lenses, $T \& R$ procedure) $(6,7,37)$.

\section{Comparative Therapies}

Topical brinzolamide had similar therapeutic effects on the INS waveforms compared to systemic acetazolamide. These benefits also mimicked the effects of the T\&R surgical procedure. Increases of $50 \%$ in both the peak NAFX and the LFD occurred, and these increases were documented within 5 hours of administration of the eyedrops. The clinical implication of the improvements shown in Figure 1 is that the subject will have a higher posttreatment peak visual acuity (increase in the peak NAFX value) and that the improved acuity will be present over a larger range of gaze angles (increase in the LFD value) than pretreatment. Only convergence provided a larger improvement in peak visual acuity (peak NAFX value) in our patient.

The percent increase curves shown in Figure 3 are used to estimate, prior to 4-muscle surgery, the percent improvement and final values for both the NAFX and the LFD and allows estimation of the improvement in measured (not just potential) visual acuity of INS patients with or without afferent visual deficits (12).

Given the positive therapeutic effects of brinzolamide eyedrops on INS, it would be preferable to an oral preparation of CAI because it has no systemic side effects and acts faster. Thus, the patient could use it on an as-needed basis.

Testing the efficacy and/or effectiveness of topical brinzolamide as a therapy for others with INS requires prospective clinical trials. The results of this preliminary study provide data to support the hypothesis that CAI eyedrops may be used as an intervention in the treatment of the nystagmus waveforms associated with INS in future randomized trials $(8,9)$.

\section{REFERENCES}

1. Dell'Osso LF, Daroff RB. Congenital nystagmus waveforms and foveation strategy. Doc Ophthalmol. 1975;39:155-182.

2. Kerrison JB, Giorda R, Lenart TD, Drack AV, Maumenee IH. Clinical and genetic analysis of a family with X-linked congenital nystagmus (NYS1). Ophthalmic Genet. 2001;22: 241-248.

3. Dell'Osso LF. Fixation characteristics in hereditary congenital nystagmus. Am J Optom Arch Am Acad Optom. 1973;50:85-90.
4. Hertle RW, Dell'Osso LF, FitzGibbon EJ, Thompson D, Yang D, Mellow SD. Horizontal rectus tenotomy in patients with congenital nystagmus. Results in 10 adults. Ophthalmology. 2003;110:2097-2105.

5. Wang Z, Dell'Osso LF, Jacobs JB, Burnstine RA, Tomsak RL. Effects of tenotomy on patients with infantile nystagmus syndrome: foveation improvement over a broadened visual field. JAAPOS. 2006;10:552-560.

6. Wang ZI, Dell'Osso LF, Tomsak RL, Jacobs JB. Combining recessions (nystagmus and strabismus) with tenotomy improved visual function and decreased oscillopsia and diplopia in acquired downbeat nystagmus and in horizontal infantile nystagmus syndrome. J AAPOS. 2007;11:135-141.

7. Taibbi G, Wang ZI, Dell'Osso LF. Infantile nystagmus syndrome: broadening the high-foveation-quality field with contact lenses. Clin Ophthalmol. 2008;2:585-590.

8. Sarvananthan N, Proudlock FA, Choudhuri I, Dua H, Gottlob I. Pharmacologic treatment of congenital nystagmus. Arch Ophthalmol. 2006;124:916-918.

9. Shery T, Proudlock FA, Sarvananthan N, McLean RJ, Gottlob I. The effects of gabapentin and memantine in acquired and congenital nystagmus: a retrospective study. $\mathrm{Br} \mathrm{J}$ Ophthalmol. 2006;90:839-843.

10. McLean R, Proudlock F, Thomas S, Degg C, Gottlob I. Congenital nystagmus: randomized, controlled, double-masked trial of memantine/gabapentin. Ann Neurol. 2007;61:130-138.

11. Serra A, Dell'Osso LF, Jacobs JB, Burnstine RA. Combined gazeangle and vergence variation in infantile nystagmus: two therapies that improve the high-visual acuity field and methods to measure it. Invest Ophthalmol Vis Sci. 2006;47:2451-2460.

12. Wang ZI, Dell'Osso LF. A review of the tenotomy nystagmus surgery: origin, mechanism, and general efficacy. NeuroOphthalmology 2007;31:157-165.

13. Thurtell MJ, Dell'Osso LF, Leigh RJ, Matta M, Jacobs JB, Tomsak RL. Effects of acetazolamide on infantile nystagmus syndrome waveforms: comparisons to contact lenses and convergence in a well-studied subject. Open Ophthalmol J. 2010;4:37-46.

14. CEMAS Working Group. A Classification of Eye Movement Abnormalities and Strabismus (CEMAS). Report of a National Eye Institute Sponsored Workshop. Bethesda, MD: National Institutes of Health, National Eye Institute, 2001.

15. Dell'Osso LF, Jacobs JB. An expanded nystagmus acuity function: intra- and intersubject prediction of best-corrected visual acuity. Doc Ophthalmol. 2002;104:249-276.

16. Dell'Osso LF. Using the NAFX for eye-movement fixation data analysis and display. OMLAB Report \#111005. 2005: 1-7. http://www.omlab.org/OMLAB_page/Teaching/ teaching.html. Accessed June 8, 2011.

17. Dell'Osso LF, Hertle RW, Williams RW, Jacobs JB. A new surgery for congenital nystagmus: effects of tenotomy on an achiasmatic canine and the role of extraocular proprioception. J AAPOS. 1999;3:166-182.

18. Hertle RW, Dell'Osso LF, FitzGibbon EJ, Yang D, Mellow SD. Horizontal rectus muscle tenotomy in children with infantile nystagmus syndrome: a pilot study. J AAPOS. 2004;8: 539-548.

19. Hertle RW, Chan C, Galita DA, Maybodi M, Crawford MA. Neuroanatomy of the extraocular muscle tendon enthesis in macaque, normal human and patients with congenital nystagmus. J AAPOS. 2002;6:319-327.

20. Büttner-Ennever JA, Horn AKE, Graf W, Ugolini G. Modern concepts of brainstem anatomy. From extraocular motoneurons to proprioceptive pathways. In: Kaminski HJ, Leigh RJ, eds. Neurobiology of Eye Movements. From Molecules to Behavior. Vol 956. New York, NY: NYAS, 2002:75-84.

21. Büttner-Ennever JA, Eberhorn A, Horn AK. Motor and sensory innervation of extraocular eye muscles. Ann N Y Acad Sci. 2003;1004:40-49.

22. Hertle RW, Anninger W, Yang D, Shatnawi R, Hill VM. Effects of extraocular muscle surgery on 15 patients with oculo-cutaneous albinism (OCA) and infantile nystagmus syndrome (INS). Am J Ophthalmol. 2004;138:978-987. 
23. Riley DA, Lang DH. Carbonic anhydrase activity of human peripheral nerves: a possible histochemical aid to nerve repair. J Hand Surg Am. 1984;9A:112-120.

24. Peyronnard JM, Messier JP, Charron L, Lavoie J, Bergouignan FX, Dubreuil M. Carbonic anhydrase activity in the normal and injured peripheral nervous system of the rat. Exp Neurol. 1986;93:481-499.

25. Fujii H, Nakamura K, Takeo K, Kawai S. Heterogeneity of carbonic anhydrase and $68 \mathrm{kDa}$ neurofilament in nerve roots analyzed by two-dimensional electrophoresis. Electrophoresis. 1993;14:1074-1078.

26. Karuman PM, Soo KC. Motor innervation of the trapezius muscle: a histochemical study. Head Neck. 1996;18: 254-258.

27. Macias MY, Lehman CT, Sanger JR, Riley DA. Myelinated sensory and alpha motor axon regeneration in peripheral nerve neuromas. Muscle Nerve. 1998;21:1748-1758.

28. Tohyama K, Ide C. Carbonic anhydrase activity in axon terminals of sensory corpuscles. Arch Histol Jpn. 1987;50: 325-333.

29. Wong V, Barrett CP, Donati EJ, Guth L. Distribution of carbonic anhydrase activity in neurons of the rat. J Comp Neurol. 1987;257:122-129.

30. Szabolcs MJ, Windisch A, Koller R, Pensch M. Axon typing of rat muscle nerves using a double staining procedure for cholinesterase and carbonic anhydrase. J Histochem Cytochem. 1991;39:1617-1625.

31. Dell'Osso LF. Nystagmus basics. Normal models that simulate dysfunction. In: Hung GK, Ciuffreda KJ, eds. Models of the Visual System. New York, NY: Kluwer Academic/Plenum Publishers, 2002:711-739.
32. Jacobs JB. An Ocular Motor System Model that Simulates Congenital Nystagmus, Including Braking and Foveating Saccades [PhD Dissertation]. In: Biomedical Engineering. Cleveland, $\mathrm{OH}$ : Case Western Reserve University, 2001: 1-357.

33. Jacobs JB, Dell'Osso LF. Congenital nystagmus: hypothesis for its genesis and complex waveforms within a behavioral ocular motor system model. J Vis. 2004;4:604-625.

34. Bennicelli J, Wright JF, Komaromy A, Jacobs JB, Hauck B, Zelenaia O, Mingozzi F, Hui D, Chung D, Rex TS, Wei Z, Qu G, Zhou S, Zeiss C, Arruda VR, Acland GM, Dell'Osso LF, High KA, Maguire AM, Bennett J. Reversal of blindness in animal models of Leber congenital amaurosis using optimized AAV2-mediated gene transfer. Mol Ther. 2008; 16:458-465.

35. Jacobs JB, Dell'Osso LF, Hertle RW, Acland GM, Bennett J. Eye movement recordings as an effectiveness indicator of gene therapy in RPE65-deficient canines: implications for the ocular motor system. Invest Ophthalmol Vis Sci. 2006; 47:2865-2875.

36. Maguire AM, Simonelli F, Pierce EA, Pugh EN Jr, Mingozzi F, Bennicelli J, Banfi S, Marshall KA, Testa F, Surace EM, Rossi S, Lyubarsky A, Arruda VR, Konkle B, Stone E, Sun J, Jacobs JB, Dell'Osso LF, Hertle RW, Ma J, Redmond TM, Zhu X, Hauck B, Zelenaia O, Shindler KS, Maguire MG, Wright JF, Volpe NJ, McDonnell JW, Auricchio A, High KA, Bennett J. Safety and efficacy of gene transfer for Leber's congenital amaurosis. N Engl J Med. 2008;358: 2240-2248.

37. Dell'Osso LF, Traccis S, Abel LA, Erzurum SI. Contact lenses and congenital nystagmus. Clin Vision Sci. 1988;3:229-232. 